\title{
Optimal LQG control over continuous fading channels *
}

\author{
Alex S. Leong* Subhrakanti Dey* Jai Anand ${ }^{* *}$ \\ * Department of Electrical and Electronic Engineering, University of \\ Melbourne, Vic.3010, Australia (e-mails: asleong@unimelb.edu.au, \\ sdey@unimelb.edu.au). \\ ** Department of Electrical Engineering, Indian Institute of \\ Technology, Kharagpur, India (e-mail: iit.kgp.jaianand@gmail.com)
}

\begin{abstract}
This paper studies the optimal control of linear systems over continuous valued fading channels. The sensor measurements are sent over a fading wireless channel to a remote controller using the analog amplify and forward technique. The controller then computes a control signal, which is transmitted over another fading channel to the actuator. Under the assumption of full channel state information (CSI) for both wireless links, we derive the optimal LQG control law. In the case where full channel state information is not available but channel statistics are available, we present the optimal linear static estimator and controller. Numerical comparisons are made between the full CSI and statistical CSI solutions.
\end{abstract}

Keywords: Fading channels, LQG control, sensor networks, stability

\section{INTRODUCTION}

In recent years there has been increasing growth in the use of wireless technologies in diverse applications such as home automation, telecommunications, and industrial monitoring and control. The challenges posed by wireless channels due to its time-varying nature are considerable, and much effort has been devoted to modelling and overcoming these effects.

One way to model the wireless channel is to regard it as a channel where packets can be received if the channel is of sufficiently good quality, and dropped if the channel is poor quality. The Kalman filtering problem with Bernoulli packet losses has been studied in Sinopoli et al. (2004), who showed the existence of a threshold, such that if the packet arrival rate is below this threshold then the expected error covariance becomes unbounded. This work has been extended in various directions such as e.g. Huang and Dey (2007); Epstein et al. (2008); Xu and Hespanha (2005); Schenato (2006). The problem of control over such packet dropping links has been studied in e.g. Sinopoli et al. (2005, 2006); Imer et al. (2006); Gupta et al. (2007), and conditions on the packet arrival rates for stability of the closed loop system has been derived. See also Schenato et al. (2007) and the references therein for a review of related work.

Another way in which one can view the wireless channel is to regard it as a continuous valued channel with time-varying channel gains, with commonly used channel models such as Rayleigh or Nakagami. Kalman filtering with continuous faded measurements has been studied in e.g. Mostofi and Murray (2005); Dey et al. (2009), which showed that under certain conditions on the fading distri-

^ This work was supported by the Australian Research Council bution the expected error covariance will always remain bounded.

In this paper we extend the work of Dey et al. (2009) to LQG control over channels with continuous faded measurements. We assume fading channels between both the sensor and controller, and between the controller and actuator/plant. Under the assumption of full channel state information (CSI) we derive the optimal control law and show that the separation principle holds. We consider both the finite horizon and infinite horizon problems. In the case where channel state information is not available but channel statistics are available, the optimal linear estimator/controller will be presented, using results from De Koning (1992).

\section{SYSTEM MODEL}

A block diagram of the model we study in this paper can be found in Fig. 1. We consider a plant

$$
x_{k+1}=A x_{k}+B u_{k}+w_{k}
$$

where $x_{k} \in \mathbb{R}^{n}$ and $u_{k} \in \mathbb{R}^{m}$. We have a sensor with measurements

$$
y_{k}=C x_{k}+v_{k}
$$

where $y_{k} \in \mathbb{R}^{l}$. The noise processes $\left\{w_{k}\right\}$ and $\left\{v_{k}\right\}$ are i.i.d. zero mean Gaussian with covariances $\Sigma_{w}>0$ and $\Sigma_{v}>0$ respectively. ${ }^{1}$

The sensor transmits this measurement over a fading channel to a remote controller using the analog amplify and forwarding technique (Gastpar and Vetterli (2003)), i.e. the sensor simply amplifies and forwards its measurement to the controller. We assume that all the measurement components are sent separately via orthogonal channels

1 For a matrix $X$, we say that $X>0$ if $X$ is positive definite, and $X \geq 0$ if $X$ is positive semi-definite. 


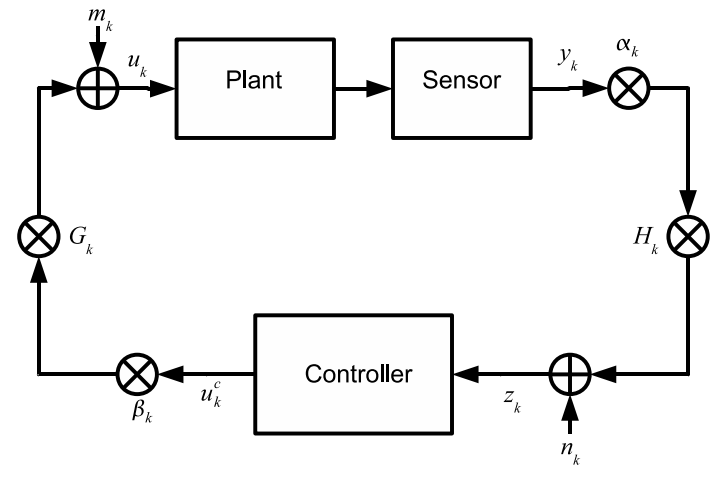

Fig. 1. System model

(Cui et al. (2007)) within the measurement time interval. The controller thus receives

$$
z_{k}=H_{k} \alpha_{k} y_{k}+n_{k}
$$

where $H_{k}=\operatorname{diag}\left(h_{k}^{1}, \ldots, h_{k}^{l}\right)$, with $h_{k}^{i} \geq 0, i=1, \ldots, l$, are the channel gains, $\alpha_{k}=\operatorname{diag}\left(\alpha_{k}^{1}, \ldots, \alpha_{k}^{l}\right)$ are the amplification factors in the analog forwarding technique, and $n_{k}$ is additive noise that represents the channel noise in the communication channel between the sensor and controller. The controller computes a control signal $u_{k}^{c} \in$ $\mathbb{R}^{m}$, which is then sent over another fading channel to the actuator/plant, again using the analog forwarding technique. The control input to the plant is thus

$$
u_{k}=G_{k} \beta_{k} u_{k}^{c}+m_{k}
$$

where $G_{k}=\operatorname{diag}\left(g_{k}^{1}, \ldots, g_{k}^{m}\right)$, with $g_{k}^{j} \geq 0, j=1, \ldots, m$, are the channel gains, $\beta_{k}=\operatorname{diag}\left(\beta_{k}^{1}, \ldots, \beta_{k}^{m}\right)$ are the amplification factors and $m_{k}$ is the channel noise between the controller and plant. The noise processes $\left\{n_{k}\right\}$ and $\left\{m_{k}\right\}$ are i.i.d. zero mean Gaussian with covariances $\Sigma_{n}$ and $\Sigma_{m}$ respectively. In this paper we assume the block fading model (see e.g. Caire et al. (1999)), such that the channels stay constant within each fading block represented by the time index $k$, but are independent from block to block. We also allow the fading processes $G_{k}$ and $H_{k}$ to have continuous distributions in general. The noise processes $w_{k}, v_{k}, n_{k}, m_{k}$ and fading processes $G_{k}, H_{k}$ are assumed to be mutually independent.

The system (1)-(4) above can be rewritten as

$$
\begin{aligned}
x_{k+1} & =A x_{k}+\bar{B}_{k} u_{k}^{c}+\bar{w}_{k} \\
z_{k} & =\bar{C}_{k} x_{k}+\bar{v}_{k}
\end{aligned}
$$

if we define $\bar{B}_{k}=B G_{k} \beta_{k}, \bar{C}_{k}=H_{k} \alpha_{k} C, \quad \bar{w}_{k}=B m_{k}+$ $w_{k}, \bar{v}_{k}=H_{k} \alpha_{k} v_{k}+n_{k}$. The noise processes $\left\{\bar{w}_{k}\right\}$ and $\left\{\bar{v}_{k}\right\}$ have covariances $\Sigma_{\bar{w}}=B \Sigma_{m} B^{T}+\Sigma_{w}$ and $\Sigma_{\bar{v}_{k}}=$ $H_{k} \alpha_{k} \Sigma_{v} \alpha_{k} H_{k}+\Sigma_{n}$ respectively.

\section{OPTIMAL LQG CONTROL UNDER FULL CSI}

We first consider the case where we have full CSI (so full knowledge of $G_{k}$ and $H_{k}$ is available to the controller at time $k$ ). The amplification factors $\alpha_{k}$ and $\beta_{k}$ are usually chosen to satisfy power constraints at the sensor transmitter and in the transmission of the control signals. Here $\alpha_{k}$ and $\beta_{k}$ are taken to be either constant or known functions of time.

\subsection{Finite horizon}

In the finite horizon case, we have a cost

$$
J_{N}=\mathbb{E}\left[x_{N}^{T} Q_{N} x_{N}+\sum_{k=0}^{N-1}\left(x_{k}^{T} Q_{k} x_{k}+u_{k}^{c^{T}} R_{k} u_{k}^{c}\right)\right]
$$

where $Q_{k} \geq 0, \forall k$ and $R_{k}>0, \forall k$. With full CSI, the information set available to the controller at time $k$ is

$$
I_{k}=\left\{z_{0}, \ldots, z_{k}, u_{0}^{c}, \ldots, u_{k-1}^{c}, H_{0}, \ldots, H_{k}, G_{0}, \ldots, G_{k}\right\}
$$

Our objective is to minimize $J_{N}$ for system (5), where the minimization is over $\left\{u_{k}^{c}\right\}$, with $u_{k}^{c}$ being a function of the information set $I_{k}$ at each time $k$.

Lemma 1. The optimal control $u_{k}^{c *}$ that minimizes $J_{N}$ in (6), subject to $u_{k}^{c}$ being a function of the information set $I_{k}$ in $(7)$, is

$$
u_{k}^{c *}=-\left(\bar{B}_{k}^{T} K_{k+1} \bar{B}_{k}+R_{k}\right)^{-1} \bar{B}_{k}^{T} K_{k+1} A \hat{x}_{k}
$$

where $\hat{x}_{k}=\mathbb{E}\left[x_{k} \mid I_{k}\right], \bar{B}_{k}=B G_{k} \beta_{k}$, and $\left\{K_{k}\right\}$ are given recursively by

$$
\begin{aligned}
K_{N}= & Q_{N}, \\
K_{k}=\mathbb{E}\left[A ^ { T } \left(K_{k+1}-K_{k+1} \bar{B}_{k}\right.\right. & \\
& \left.\left.\times\left(R_{k}+\bar{B}_{k}^{T} K_{k+1} \bar{B}_{k}\right)^{-1} \bar{B}_{k}^{T} K_{k+1}\right) A\right]+Q_{k} .
\end{aligned}
$$

The expectation in (9) is with respect to $G_{k}$ (since $\bar{B}_{k}=$ $\left.B G_{k} \beta_{k}\right)$.

Proof The proof uses dynamic programming and is along similar lines to e.g. Bertsekas (2000), see also Imer et al. (2006). Define

$$
\begin{aligned}
V_{N}\left(I_{N}\right) & =\mathbb{E}\left[x_{N}^{T} Q_{N} x_{N} \mid I_{N}\right], \\
V_{k}\left(I_{k}\right) & =\min _{u_{k}^{c}} \mathbb{E}\left[x_{k}^{T} Q_{k} x_{k}+u_{k}^{c^{T}} R_{k} u_{k}^{c}+V_{k+1}\left(I_{k+1}\right) \mid I_{k}\right]
\end{aligned}
$$

We have

$$
\begin{aligned}
V_{N-1} & \left(I_{N-1}\right) \\
= & \min _{u_{N-1}^{c}} \mathbb{E}\left\{x_{N-1}^{T} Q_{N-1} x_{N-1}+u_{N-1}^{c^{T}} R_{N-1} u_{N-1}^{c}\right. \\
& +\left(A x_{N-1}+\bar{B}_{N-1} u_{N-1}^{c}+\bar{w}_{N-1}\right)^{T} Q_{N} \\
& \times\left(A x_{N-1}+\bar{B}_{N-1} u_{N-1}^{c}+\bar{w}_{N-1} \mid I_{N-1}\right\} \\
= & \mathbb{E} \\
& \left\{x_{N-1}^{T}\left(A^{T} Q_{N} A+Q_{N-1}\right) x_{N-1} \mid I_{N-1}\right\} \\
& +\mathbb{E}\left\{\bar{w}_{N-1}^{T} Q_{N} \bar{w}_{N-1}\right\} \\
& +\min _{u_{N-1}^{c}}\left\{u_{N-1}^{c^{T}}\left(\bar{B}_{N-1}^{T} Q_{N} \bar{B}_{N-1}+R_{N-1}\right) u_{N-1}^{c}\right. \\
& \left.+2 \mathbb{E}\left[x_{N-1}^{T} \mid I_{N-1}\right] A^{T} Q_{N} \bar{B}_{N-1} u_{N-1}^{c}\right\}
\end{aligned}
$$

which gives

$$
u_{N-1}^{c *}=-\left(\bar{B}_{N-1}^{T} Q_{N} \bar{B}_{N-1}+R_{N-1}\right)^{-1} \bar{B}_{N-1}^{T} Q_{N} A \hat{x}_{N-1}
$$

Substituting back into the expression for $V_{N-1}\left(I_{N-1}\right)$, we obtain

$$
\begin{aligned}
& V_{N-1}\left(I_{N-1}\right)=\mathbb{E}\left\{\bar{w}_{N-1}^{T} Q_{N} \bar{w}_{N-1}\right\} \\
& \quad+\mathbb{E}\left\{\left(x_{N-1}-\hat{x}_{N-1}\right)^{T} \tilde{P}_{N-1}\left(x_{N-1}-\hat{x}_{N-1}\right) \mid I_{N-1}\right\} \\
& \quad+\mathbb{E}\left\{x_{N-1}^{T} \tilde{K}_{N-1} x_{N-1} \mid I_{N-1}\right\}
\end{aligned}
$$

where

$$
\begin{aligned}
\tilde{P}_{N-1} & =A^{T} Q_{N} \bar{B}_{N-1}\left(R_{N-1}+\bar{B}_{N-1}^{T} Q_{N} \bar{B}_{N-1}\right)^{-1} \bar{B}_{N-1}^{T} Q_{N} A, \\
\tilde{K}_{N-1} & =A^{T} Q_{N} A+Q_{N-1}-\tilde{P}_{N-1}
\end{aligned}
$$

For period $N-2$ we have 


$$
\begin{aligned}
& V_{N-2}\left(I_{N-2}\right) \\
& =\min _{u_{N-2}^{c}} \mathbb{E}\left\{x_{N-2}^{T} Q_{N-2} x_{N-2}\right. \\
& \left.\quad+u_{N-2}^{c^{T}} R_{N-2} u_{N-2}^{c}+V_{N-1}\left(I_{N-1}\right) \mid I_{N-2}\right\} \\
& =\mathbb{E}\left\{x_{N-2}^{T} Q_{N-2} x_{N-2} \mid I_{N-2}\right\} \\
& \quad+\min _{u_{N-2}^{c}}\left[u_{N-2}^{c^{T}} R_{N-2} u_{N-2}^{c}+\mathbb{E}\left\{x_{N-1}^{T} \tilde{K}_{N-1} x_{N-1} \mid I_{N-2}\right\}\right] \\
& \quad+\mathbb{E}\left\{\left(x_{N-1}-\hat{x}_{N-1}\right)^{T} P_{N-1}\left(x_{N-1}-\hat{x}_{N-1}\right) \mid I_{N-2}\right\} \\
& \quad+\mathbb{E}\left\{\bar{w}_{N-1}^{T} Q_{N} \bar{w}_{N-1}\right\}
\end{aligned}
$$

Now

$$
\begin{aligned}
& \min _{u_{N-2}^{c}}\left[u_{N-2}^{c^{T}} R_{N-2} u_{N-2}^{c}+\mathbb{E}\left\{x_{N-1}^{T} \tilde{K}_{N-1} x_{N-1} \mid I_{N-2}\right\}\right] \\
& =\min _{u_{N-2}^{c}}\left[u_{N-2}^{c^{T}} R_{N-2} u_{N-2}^{c}\right. \\
& \quad+\mathbb{E}\left\{\left(A x_{N-2}+\bar{B}_{N-2} u_{N-2}^{c}+\bar{w}_{N-2}\right)^{T} \tilde{K}_{N-1}\right. \\
& \left.\left.\quad \times\left(A x_{N-2}+\bar{B}_{N-2} u_{N-2}^{c}+\bar{w}_{N-2}\right) \mid I_{N-2}\right\}\right] \\
& =\min _{u_{N-2}^{c}}\left\{u_{N-2}^{c^{T}} R_{N-2} u_{N-2}^{c}+u_{N-2}^{c^{T}} \bar{B}_{N-2}^{T} \mathbb{E}\left[\tilde{K}_{N-1}\right] \bar{B}_{N-2} u_{N-2}^{c}\right. \\
& \left.\quad+2 u_{N-2}^{c^{T}} \bar{B}_{N-2}^{T} \mathbb{E}\left[\tilde{K}_{N-1}\right] A \hat{x}_{N-2}\right\} \\
& \quad+\mathbb{E}\left[x_{N-2}^{T} A^{T} \tilde{K}_{N-1} A x_{N-2} \mid I_{N-2}\right]+\mathbb{E}\left[\bar{w}_{N-2}^{T} \tilde{K}_{N-1} \bar{w}_{N-2}\right]
\end{aligned}
$$

and so

$$
\begin{aligned}
u_{N-2}^{c *}=- & \left(\bar{B}_{N-2}^{T} \mathbb{E}\left[\tilde{K}_{N-1}\right] \bar{B}_{N-2}+R_{N-2}\right)^{-1} \\
& \times \bar{B}_{N-2}^{T} \mathbb{E}\left[\tilde{K}_{N-1}\right] A \hat{x}_{N-2}
\end{aligned}
$$

Continuing on, we will obtain

$$
u_{k}^{c *}=-\left(\bar{B}_{k}^{T} K_{k+1} \bar{B}_{k}+R_{k}\right)^{-1} \bar{B}_{k}^{T} K_{k+1} A \hat{x}_{k}
$$

where $K_{k}$ are given by

$$
\begin{aligned}
K_{N}= & Q_{N}, \\
K_{k}=\mathbb{E} & {\left[A ^ { T } \left(K_{k+1}-K_{k+1} \bar{B}_{k}\right.\right.} \\
& \left.\left.\times\left(R_{k}+\bar{B}_{k}^{T} K_{k+1} \bar{B}_{k}\right)^{-1} \bar{B}_{k}^{T} K_{k+1}\right) A\right]+Q_{k} .
\end{aligned}
$$

The optimal control $u_{k}^{c *}$ in (8) is a linear function of $\hat{x}_{k}=\mathbb{E}\left[x_{k} \mid I_{k}\right]$, which can be computed with the standard time-varying Kalman filter. Hence a separation principle holds for this problem. Further define

$$
\begin{aligned}
\hat{x}_{k+1 \mid k} & =\mathbb{E}\left[x_{k+1} \mid I_{k}\right] \\
P_{k+1 \mid k} & =\mathbb{E}\left[\left(x_{k+1}-\hat{x}_{k+1 \mid k}\right)\left(x_{k+1}-\hat{x}_{k+1 \mid k}\right)^{T} \mid I_{k}\right] \\
P_{k \mid k} & =\mathbb{E}\left[\left(x_{k}-\hat{x}_{k \mid k}\right)\left(x_{k}-\hat{x}_{k \mid k}\right)^{T} \mid I_{k}\right]
\end{aligned}
$$

Below, we summarise the Kalman filtering equations:

$$
\begin{aligned}
\hat{x}_{k} & =\hat{x}_{k \mid k-1}+S_{k}\left(z_{k}-\bar{C}_{k} \hat{x}_{k \mid k-1}\right) \\
\hat{x}_{k \mid k-1} & =A \hat{x}_{k-1}+\bar{B}_{k-1} u_{k-1}^{c} \\
S_{k} & =P_{k \mid k-1} \bar{C}_{k}^{T}\left(\bar{C}_{k} P_{k \mid k-1} \bar{C}_{k}^{T}+\Sigma_{\bar{v}_{k}}\right)^{-1} \\
P_{k \mid k} & =P_{k \mid k-1}-S_{k} \bar{C}_{k} P_{k \mid k-1} \\
P_{k \mid k-1} & =A P_{k-1 \mid k-1} A^{T}+\Sigma_{\bar{w}}
\end{aligned}
$$

where $\bar{B}_{k}=B G_{k} \beta_{k}, \bar{C}_{k}=H_{k} \alpha_{k} C, \Sigma_{\bar{w}}=B \Sigma_{m} B^{T}+\Sigma_{w}$ and $\Sigma_{\bar{v}_{k}}=H_{k} \alpha_{k} \Sigma_{v} \alpha_{k} H_{k}+\Sigma_{n}$.

Remark: The expectation in (9) is in general difficult to compute analytically, due to the difficulty in explicitly evaluating the expectation of the nonlinear term. However in cases such as scalar control signals, closed form expressions can be obtained for specific fading distributions. See Section 5 for an example with Rayleigh fading.
Remark: If the fading process $\left\{G_{k}\right\}$ is discrete (i.e. components of $G_{k}$ take on discrete values), then the optimal controller can also be derived by using results on optimal control of jump linear systems, see Chizeck and Ji (1988).

Remark: System (5) is a time-varying linear system. If we attempt to apply the standard solution of the time-varying LQG problem to (5) directly, we obtain

$$
u_{k}^{c}=-\left(R_{k}+\bar{B}_{k}^{T} K_{k+1} \bar{B}_{k}\right)^{-1} \bar{B}_{k}^{T} K_{k+1} A \hat{x}_{k \mid k}
$$

where $K_{k}$ are given by

$$
\begin{aligned}
K_{N}= & Q_{N}, \\
K_{k}= & A^{T}\left(K_{k+1}-K_{k+1} \bar{B}_{k}\right. \\
& \left.\times\left(R_{k}+\bar{B}_{k}^{T} K_{k+1} \bar{B}_{k}\right)^{-1} \bar{B}_{k}^{T} K_{k+1}\right) A+Q_{k} .
\end{aligned}
$$

However, the recursions given by (11) are non-causal since $K_{k}$ requires knowledge of $G_{k+j}, j=1,2, \ldots$, and so $u_{k}^{c}$ given by (10) is not a function of the information set $I_{k}$ in (7). We will however use this non-causal solution in numerical comparisons with the optimal causal solution in Section 5 .

\subsection{Infinite horizon}

In the infinite horizon case we take $\alpha_{k}=\alpha, \beta_{k}=\beta, Q_{k}=$ $Q>0, R_{k}=R>0, \forall k{ }^{2}$ We will assume that the pairs $(A, B)$ and $\left(A, \Sigma_{w}^{1 / 2}\right)$ are stabilizable, and the pairs $(A, C)$ and $\left(A, Q^{1 / 2}\right)$ are detectable. We now have a cost function

$$
J_{\infty}=\lim _{N \rightarrow \infty} \frac{J_{N}}{N}=\lim _{N \rightarrow \infty} \frac{1}{N} \mathbb{E}\left[\sum_{k=0}^{N-1}\left(x_{k}^{T} Q x_{k}+u_{k}^{c^{T}} R u_{k}^{c}\right)\right]
$$

We have the following result:

Lemma 2. Assume that $\alpha$ and $\beta$ are invertible, the components of $G_{k}$ have continuous distributions such that $\operatorname{Pr}\left(g_{k}^{i}>0\right)=1, \forall k, i$, and the components of $H_{k}$ have continuous distributions such that $\operatorname{Pr}\left(h_{k}^{j}>0\right)=$ $1, \forall k, j$. Furthermore assume that $A$ is invertible and that $\max \left(0, \log \left\|H_{0} C\right\|\right)$ is integrable. Then

(i) The expected error covariance $\mathbb{E}\left[P_{k \mid k}\right]$ remains bounded as $k \rightarrow \infty$.

(ii) The optimal cost $J_{\infty}^{*}$ is finite, and the optimal control $u_{k}^{c *}$ that minimizes $J_{\infty}^{\infty}$ in (12), subject to $u_{k}^{c}$ being a function of the information set $I_{k}$ in $(7)$, is

$$
u_{k}^{c *}=-\left(\bar{B}_{k}^{T} K \bar{B}_{k}+R\right)^{-1} \bar{B}_{k}^{T} K A \hat{x}_{k}
$$

where $\hat{x}_{k}=\mathbb{E}\left[x_{k} \mid I_{k}\right]$, and $K$ is the unique solution of the fixed point equation

$$
K=\mathbb{E}\left[A^{T}\left(K-K \bar{B}_{k}\left(R+\bar{B}_{k}^{T} K \bar{B}_{k}\right)^{-1} \bar{B}_{k}^{T} K\right) A\right]+Q
$$

\section{Proof}

(i) Under the assumptions of Lemma 2, the boundedness of the expected error covariance $\mathbb{E}\left[P_{k+1 \mid k}\right]$ (and hence $\left.\mathbb{E}\left[P_{k \mid k}\right]\right)$ for Kalman filtering with faded measurements (and no control) has previously been shown in Dey et al. (2009). Noting that the Kalman filtering recursions for $P_{k \mid k}$ do not depend on the control signals $u_{k}^{c}$ (Anderson and Moore, 1979, p.110), the result follows.

(ii) We first show that the optimal control takes the form (13). From the finite horizon recursions (9) and reversing

\footnotetext{
2 For instance, the case $\alpha_{k}=1, \beta_{k}=1$ would correspond to direct forwarding of the sensor measurements and control signals without any scaling.
} 
the time index as in Imer et al. (2006), we have the recursion

$$
\begin{gathered}
\dot{K}_{k+1}=\mathbb{E}\left[A ^ { T } \left(\dot{K}_{k}-\dot{K}_{k} B G \beta\left(R+\beta G B^{T} \dot{K}_{k} B G \beta\right)^{-1}\right.\right. \\
\left.\left.\times \beta G B^{T} \dot{K}_{k}\right) A\right]+Q
\end{gathered}
$$

Under our stabilizability and detectability assumptions, it can be shown that as $k \rightarrow \infty, \dot{K}_{k}$ converges to the unique fixed point of the equation

$K=\mathbb{E}\left[A^{T}\left(K-K B G \beta\left(R+\beta G B^{T} K B G \beta\right)^{-1} \beta G B^{T} K\right) A\right]+Q$ by using a similar proof to Theorem 3.3 of Dey et al. (2009). Taking the limit $N \rightarrow \infty$ of the solution to the finite horizon problem then gives the desired result.

We now show that the optimal cost $J_{\infty}^{*}$ is finite. Let us call $L_{k}=-\left(\bar{B}_{k}^{T} K \bar{B}_{k}+R\right)^{-1} \bar{B}_{k}^{T} K A$ and $e_{k}=x_{k}-\hat{x}_{k}$. Noting that $\mathbb{E}\left[\hat{x}_{k} e_{k}^{T}\right]=\mathbb{E}\left[e_{k} \hat{x}_{k}^{T}\right]=0$, we have

$$
\begin{aligned}
& \frac{1}{N} \mathbb{E}\left[\sum_{k=0}^{N-1}\left(x_{k}^{T} Q x_{k}+u_{k}^{c *^{T}} R u_{k}^{c *}\right)\right] \\
= & \frac{1}{N} \mathbb{E}\left[\sum_{k=0}^{N-1}\left(x_{k}^{T} Q x_{k}+\hat{x}_{k}^{T} L_{k}^{T} R L_{k} \hat{x}_{k}\right)\right] \\
= & \frac{1}{N} \mathbb{E}\left[\sum_{k=0}^{N-1}\left(x_{k}^{T}\left(Q+L_{k}^{T} R L_{k}\right) x_{k}-e_{k}^{T} L_{k}^{T} R L_{k} e_{k}\right)\right] \\
= & \frac{1}{N} \mathbb{E}\left[\sum_{k=0}^{N-1}\left[\operatorname{Tr}\left(\left(Q+L_{k}^{T} R L_{k}\right) x_{k} x_{k}^{T}\right)-\operatorname{Tr}\left(L_{k}^{T} R L_{k} e_{k} e_{k}^{T}\right)\right]\right. \\
= & \frac{1}{N} \sum_{k=0}^{N-1} \operatorname{Tr}\left(\mathbb{E}\left(Q+L_{k}^{T} R L_{k}\right) \mathbb{E}\left(x_{k} x_{k}^{T}\right)\right) \\
& -\frac{1}{N} \sum_{k=0}^{N-1} \operatorname{Tr}\left(\mathbb{E}\left(L_{k}^{T} R L_{k}\right) \mathbb{E}\left(P_{k \mid k}\right)\right)
\end{aligned}
$$

where the last line holds since $x_{k}$ does not depend on $G_{k}$ (and hence $L_{k}$ ). The second term above remains bounded as $N \rightarrow \infty$ by part (i). The first term above will also be bounded as $N \rightarrow \infty$ if we can show $\mathbb{E}\left(x_{k} x_{k}^{T}\right)$ is bounded for the system

$x_{k+1}=A x_{k}+\bar{B}_{k} L_{k} \hat{x}_{k}+\bar{w}_{k}=\left(A+\bar{B}_{k} L_{k}\right) x_{k}-\bar{B}_{k} L_{k} e_{k}+\bar{w}_{k}$ By similar arguments as in Imer et al. (2006), this is true if and only if the system

$$
\xi_{k+1}=\left(A+\bar{B}_{k} L_{k}\right) \xi_{k}
$$

is mean square stable. We can verify that

$$
\begin{aligned}
& \left(A+\bar{B}_{k} L_{k}\right)^{T} K\left(A+\bar{B}_{k} L_{k}\right)+L_{k}^{T} R L_{k}+Q \\
& \quad=A^{T} K A-A^{T} K^{T} \bar{B}_{k}\left(\bar{B}_{k}^{T} K \bar{B}_{k}+R\right)^{-1} \bar{B}_{k}^{T} K A+Q
\end{aligned}
$$

and so

$$
K=\mathbb{E}\left[\left(A+\bar{B}_{k} L_{k}\right)^{T} K\left(A+\bar{B}_{k} L_{k}\right)+L_{k}^{T} R L_{k}+Q\right]
$$

Hence

$$
\begin{aligned}
\mathbb{E} & {\left[\xi_{k+1}^{T} K \xi_{k+1}-\xi_{k} K \xi_{k}\right] } \\
& =\mathbb{E}\left[\xi_{k}^{T}\left(\left(A+\bar{B}_{k} L_{k}\right)^{T} K\left(A+\bar{B}_{k} L_{k}\right)-K\right) \xi_{k}\right] \\
& =-\mathbb{E}\left[\xi_{k}^{T} \mathbb{E}\left(L_{k}^{T} R L_{k}+Q\right) \xi_{k}\right]
\end{aligned}
$$

where the last line holds since $\xi_{k}$ does not depend on $G_{k}$. Therefore

$$
\mathbb{E}\left[\xi_{k+1}^{T} K \xi_{k+1}\right]=\mathbb{E}\left[\xi_{0}^{T} K \xi_{0}\right]-\sum_{i=0}^{k} \mathbb{E}\left[\xi_{i}^{T} \mathbb{E}\left(L_{i}^{T} R L_{i}+Q\right) \xi_{i}\right]
$$

Using similar arguments to Imer et al. (2006) (see also Bertsekas $(2000))$, we can then show that $\mathbb{E}\left[\xi_{k}^{T} \xi_{k}\right] \rightarrow 0$ as $k \rightarrow \infty$.

Thus for any fading processes $G_{k}$ and $H_{k}$ satisfying the conditions of Lemma 2, the problem of minimizing (12) is well defined, and the minimum $J_{\infty}$ will be finite.

\section{OPTIMAL LINEAR CONTROL WITH STATISTICAL CSI}

In this section we consider the case where we don't have knowledge of the values $G_{k}$ and $H_{k}$ (e.g. either because it is too difficult or requires too many resources to obtain), but know their channel statistics. By similar arguments to Schenato et al. (2007), the optimal controller can be shown to be generally nonlinear and difficult to derive. One alternative is to derive the optimal linear controller and estimator. Here we will use a static linear estimator and controller of the form

$$
\begin{aligned}
\hat{x}_{k+1} & =F \hat{x}_{k}+K z_{k} \\
u_{k}^{c} & =-L \hat{x}_{k}
\end{aligned}
$$

We again consider the infinite horizon case where we take $\alpha_{k}=\alpha, \beta_{k}=\beta, Q_{k}=Q>0, R_{k}=R>0, \forall k$, and assume that the pairs $(A, B)$ and $\left(A, \Sigma_{w}^{1 / 2}\right)$ are stabilizable, and the pairs $(A, C)$ and $(A, Q)$ are detectable. The cost function that we wish to minimize is the infinite horizon cost

$$
J_{\infty}=\lim _{N \rightarrow \infty} \frac{1}{N} \mathbb{E}\left[\sum_{k=0}^{N-1}\left(x_{k}^{T} Q x_{k}+u_{k}^{c^{T}} R u_{k}^{c}\right)\right]
$$

where the minimization is over $(F, K, L)$. The situation above falls within the framework of systems with white parameters. In De Koning (1992) necessary and sufficient conditions for minimizing (16) subject to the estimator and controller being of the form (15) is derived, using techniques such as the matrix minimum principle. A method for computing the optimal $F, K$ and $L$ is then also given. Below we will present the method of De Koning (1992) adapted to our situation. ${ }^{3}$

Define the following recursions:

$$
\begin{aligned}
& X_{1, k+1}=A^{T} X_{1, k} A-L_{k}^{T}\left(\mathbb{E}\left[\bar{B}_{k}^{T} X_{1, k} \bar{B}_{k}\right]+R\right. \\
& \left.\quad+\mathbb{E}\left[\bar{B}_{k}^{T} X_{2, k} \bar{B}_{k}\right]-\mathbb{E}\left[\bar{B}_{k}^{T}\right] X_{2, k} \mathbb{E}\left[\bar{B}_{k}\right]\right) L_{k} \\
& \quad+Q+\mathbb{E}\left[\bar{C}_{k}^{T} K_{k}^{T} X_{2, k} K_{k} \bar{C}_{k}\right]-\mathbb{E}\left[\bar{C}_{k}^{T}\right] K_{k}^{T} X_{2, k} K_{k} \mathbb{E}\left[\bar{C}_{k}\right] \\
& \quad X_{2, k+1}=\left(A-K_{k} \mathbb{E}\left[\bar{C}_{k}\right]\right)^{T} X_{2, k}\left(A-K_{k} \mathbb{E}\left[\bar{C}_{k}\right]\right) \\
& \quad+L_{k}^{T}\left(\mathbb{E}\left[\bar{B}_{k}^{T} X_{1, k} \bar{B}_{k}\right]+R\right. \\
& \left.\quad+\mathbb{E}\left[\bar{B}_{k}^{T} X_{2, k} \bar{B}_{k}\right]-\mathbb{E}\left[\bar{B}_{k}^{T}\right] X_{2, k} \mathbb{E}\left[\bar{B}_{k}\right]\right) L_{k} \\
& X_{3, k+1}=A X_{3, k} A^{T}-K_{k}\left(\mathbb{E}\left[\bar{C}_{k} X_{3, k} \bar{C}_{k}^{T}\right]+\mathbb{E}\left[\Sigma_{\bar{v}_{k}}\right]\right. \\
& \left.\quad+\mathbb{E}\left[\bar{C}_{k} X_{4, k} \bar{C}_{k}^{T}\right]-\mathbb{E}\left[\bar{C}_{k}\right] X_{4, k} \mathbb{E}\left[\bar{C}_{k}^{T}\right]\right) K_{k}^{T} \\
& \quad+\Sigma_{\bar{w}}+\mathbb{E}\left[\bar{B}_{k} L_{k} X_{4, k} L_{k}^{T} \bar{B}_{k}^{T}\right]-\mathbb{E}\left[\bar{B}_{k}\right] L_{k} X_{4, k} L_{k}^{T} \mathbb{E}\left[\bar{B}_{k}^{T}\right] \\
& X_{4, k+1}=\left(A-\mathbb{E}\left[\bar{B}_{k}\right] L_{k}\right) X_{4, k}\left(A-\mathbb{E}\left[\bar{B}_{k}\right] L_{k}\right)^{T} \\
& \quad+K_{k}\left(\mathbb{E}\left[\bar{C}_{k} X_{3, k} \bar{C}_{k}^{T}\right]+\mathbb{E}\left[\Sigma_{\bar{v}_{k}}\right]+\mathbb{E}\left[\bar{C}_{k} X_{4, k} \bar{C}_{k}^{T}\right]\right. \\
& \left.\quad-\mathbb{E}\left[\bar{C}_{k}\right] X_{4, k} \mathbb{E}\left[\bar{C}_{k}^{T}\right]\right) K_{k}^{T}
\end{aligned}
$$

\footnotetext{
3 Note that in our situation $\Sigma_{\bar{v}_{k}}$ is time-varying, however it can be verified that the role of $W$ in De Koning (1992) can be replaced by $\mathbb{E}\left[\Sigma_{\bar{v}_{k}}\right]$ here.
} 


$$
\begin{aligned}
& F_{k+1}=A-\mathbb{E}\left[\bar{B}_{k}\right] L_{k}-K_{k} \mathbb{E}\left[\bar{C}_{k}\right] \\
& K_{k+1}=A X_{3, k} \mathbb{E}\left[\bar{C}_{k}^{T}\right]\left(\mathbb{E}\left[\bar{C}_{k} X_{3, k} \bar{C}_{k}^{T}\right]+\mathbb{E}\left[\Sigma_{\bar{v}_{k}}\right]\right. \\
& \left.\quad+\mathbb{E}\left[\bar{C}_{k} X_{4, k} \bar{C}_{k}^{T}\right]-\mathbb{E}\left[\bar{C}_{k}\right] X_{4, k} \mathbb{E}\left[\bar{C}_{k}^{T}\right]\right)^{\dagger} \\
& L_{k+1}=\left(\mathbb{E}\left[\bar{B}_{k}^{T} X_{1, k} \bar{B}_{k}\right]+R+\mathbb{E}\left[\bar{B}_{k}^{T} X_{2, k} \bar{B}_{k}\right]\right. \\
& \left.\quad-\mathbb{E}\left[\bar{B}_{k}^{T}\right] X_{2, k} \mathbb{E}\left[\bar{B}_{k}\right]\right)^{\dagger} \mathbb{E}\left[\bar{B}_{k}^{T}\right] X_{1, k} A
\end{aligned}
$$

where $\dagger$ represents the Moore-Penrose inverse. We also have the concept of mean square compensatability introduced in De Koning (1992).

Definition: We say that $\left(A, \bar{B}_{k}, \bar{C}_{k}\right)$ is mean square compensatable if there exist $F, K, L$ such that the system

$$
x_{k+1}^{\prime}=\Phi_{k}^{\prime} x_{k}^{\prime}
$$

has $\mathbb{E}\left[\left\|x_{k}^{\prime}\right\|^{2}\right] \rightarrow 0$ as $k \rightarrow \infty$, where

$$
\Phi_{k}^{\prime}=\left[\begin{array}{cc}
A & -\bar{B}_{k} L \\
K \bar{C}_{k} & F
\end{array}\right] .
$$

We then have the following:

Lemma 3. (i) Assume that $\left(A, \bar{B}_{k}, \bar{C}_{k}\right)$ is mean square compensatable. Then starting from $X_{1,0}=0, X_{2,0}=$ $0, X_{3,0}=0, X_{4,0}=0$ (where 0 here represents the zero matrix), the recursions (17)-(18) converge to limiting values as $k \rightarrow \infty$. The optimal $F^{*}, K^{*}, L^{*}$ for (15) are given by the limiting values of the $F_{k}, K_{k}, L_{k}$ recursions respectively.

(ii) $\left(A, \bar{B}_{k}, \bar{C}_{k}\right)$ is mean square compensatable if and only if the recursions (17)-(18) converge to limiting values as $k \rightarrow \infty$.

Proof (i) This is essentially Theorem 3 of De Koning (1992).

(ii) See Theorem 4 of De Koning (1992).

The expectations involved in (17)-(18) can usually be computed without difficulty. For instance, we have $\mathbb{E}\left[\bar{B}_{k}\right]=$ $B \mathbb{E}\left[G_{k}\right] \beta=B \operatorname{diag}\left(\mathbb{E}\left[g_{1}\right], \ldots, \mathbb{E}\left[g_{m}\right]\right) \beta$, and $\mathbb{E}\left[\bar{C}_{k}\right]=$ $\operatorname{diag}\left(\mathbb{E}\left[h_{1}\right], \ldots, \mathbb{E}\left[h_{l}\right]\right) \alpha C$. Next call

$$
\Gamma=\left[\begin{array}{cccc}
\mathbb{E}\left[g_{1}^{2}\right] & \mathbb{E}\left[g_{1}\right] \mathbb{E}\left[g_{2}\right] & \ldots & \mathbb{E}\left[g_{1}\right] \mathbb{E}\left[g_{m}\right] \\
\mathbb{E}\left[g_{2}\right] \mathbb{E}\left[g_{1}\right] & \mathbb{E}\left[g_{2}^{2}\right] & \ldots & \mathbb{E}\left[g_{2}\right] \mathbb{E}\left[g_{m}\right] \\
\vdots & \vdots & \ddots & \vdots \\
\mathbb{E}\left[g_{m}\right] \mathbb{E}\left[g_{1}\right] & \mathbb{E}\left[g_{m}\right] \mathbb{E}\left[g_{2}\right] & \ldots & \mathbb{E}\left[g_{m}^{2}\right]
\end{array}\right]
$$

Then note that $\mathbb{E}[G X G]=\Gamma \circ X$, where $\circ$ is the Hadamard or element-wise product (Horn and Johnson (1991)). Hence $\mathbb{E}\left[\bar{B}_{k} X \bar{B}_{k}^{T}\right]=B \mathbb{E}[G \beta X \beta G] B^{T}=B(\Gamma \circ$ $(\beta X \beta)) B^{T}$ and $\mathbb{E}\left[\bar{B}_{k}^{T} X \bar{B}_{k}\right]=\beta\left(\Gamma \circ\left(B^{T} X B\right)\right) \beta$. Similarly, if we call

$$
\Lambda=\left[\begin{array}{cccc}
\mathbb{E}\left[h_{1}^{2}\right] & \mathbb{E}\left[h_{1}\right] \mathbb{E}\left[h_{2}\right] & \ldots & \mathbb{E}\left[h_{1}\right] \mathbb{E}\left[h_{l}\right] \\
\mathbb{E}\left[h_{2}\right] \mathbb{E}\left[h_{1}\right] & \mathbb{E}\left[h_{2}^{2}\right] & \ldots & \mathbb{E}\left[h_{2}\right] \mathbb{E}\left[h_{l}\right] \\
\vdots & \vdots & \ddots & \vdots \\
\mathbb{E}\left[h_{l}\right] \mathbb{E}\left[h_{1}\right] & \mathbb{E}\left[h_{l}\right] \mathbb{E}\left[h_{2}\right] & \ldots & \mathbb{E}\left[h_{l}^{2}\right]
\end{array}\right],
$$

then $\mathbb{E}\left[\bar{C}_{k} X \bar{C}_{k}^{T}\right]=\Lambda \circ\left(\alpha C X C^{T} \alpha\right)$ and $\mathbb{E}\left[\bar{C}_{k}^{T} X \bar{C}_{k}\right]=$ $C^{T} \alpha(\Lambda \circ X) \alpha C$. We also have $\mathbb{E}\left[\Sigma_{\bar{v}_{k}}\right]=\Lambda \circ\left(\alpha \Sigma_{v} \alpha\right)+\Sigma_{n}$.

Remark: The stability criteria of Lemma 3 (ii) involves checking if $\left(X_{1, k}, X_{2, k}, X_{3, k}, X_{4, k}\right)$ converges as $k \rightarrow \infty$ in the recursion (17)-(18). Determining whether the recursions converge can be achieved via numerical computation as described above, however analytical criteria seem to be more complicated to obtain.

\section{NUMERICAL EXAMPLE}

We consider a scalar system, with $g_{k}$ and $h_{k}$ both Rayleigh distributed, so that $g_{k}^{2}$ and $h_{k}^{2}$ are exponentially distributed with means $1 / \lambda_{g}$ and $1 / \lambda_{h}$ respectively.

The optimal control in the case of full CSI is then

with

$$
u_{k}^{c *}=\frac{-g_{k} a K_{k+1} \beta_{k} b}{g_{k}^{2} \beta_{k}^{2} b^{2} K_{k+1}+R_{k}} \hat{x}_{k}
$$

$$
\begin{aligned}
K_{N} & =Q_{N} \\
K_{k} & =\mathbb{E}\left[\frac{a^{2} K_{k+1} R_{k}}{g_{k}^{2} \beta_{k}^{2} b^{2} K_{k+1}+R_{k}}\right]+Q_{k} \\
& =\frac{\lambda_{g} a^{2} R_{k}}{\beta_{k}^{2} b^{2}} \exp \left(\frac{\lambda_{g} R_{k}}{\beta_{k}^{2} b^{2} K_{k+1}}\right) E_{1}\left(\frac{\lambda_{g} R_{k}}{\beta_{k}^{2} b^{2} K_{k+1}}\right)+Q_{k}
\end{aligned}
$$

where $E_{1}(x)$ is the exponential integral.

In the computation of the optimal linear controller in the case with statistical CSI, the terms in the recursions simplify to $\mathbb{E}\left[\bar{B}_{k}\right]=\beta b \sqrt{\frac{\pi}{4 \lambda_{g}}}, \mathbb{E}\left[\bar{C}_{k}\right]=\alpha c \sqrt{\frac{\pi}{4 \lambda_{h}}}, \Sigma_{\bar{w}}=b^{2} \sigma_{m}^{2}+$ $\sigma_{w}^{2}, \mathbb{E}\left[\Sigma_{\bar{v}_{k}}\right]=\frac{\alpha^{2} \sigma_{v}^{2}}{\lambda_{h}}+\sigma_{n}^{2}, \mathbb{E}\left[\bar{B}_{k}^{T} X \bar{B}_{k}\right]=\mathbb{E}\left[\bar{B}_{k} X \bar{B}_{k}^{T}\right]=$ $\frac{\beta^{2} b^{2}}{\lambda_{g}} X, \mathbb{E}\left[\bar{C}_{k}^{T} X \bar{C}_{k}\right]=\mathbb{E}\left[\bar{C}_{k} X \bar{C}_{k}^{T}\right]=\frac{\alpha^{2} c^{2}}{\lambda_{h}} X$.

We will consider a case with $b=c=1, \sigma_{w}^{2}=\sigma_{v}^{2}=\sigma_{n}^{2}=$ $\sigma_{m}^{2}=1, \alpha=\beta=1, Q=R=1, \lambda_{g}=2, \lambda_{h}=5$. In Figure 2 we plot the finite horizon expected cost $J_{N}$ for horizon $N=10$, and various values of $a$. We compare between the causal control given by (8)-(9), and the noncausal control given by (10)-(11). The causal solution can be seen to perform quite closely to the non-causal solution.

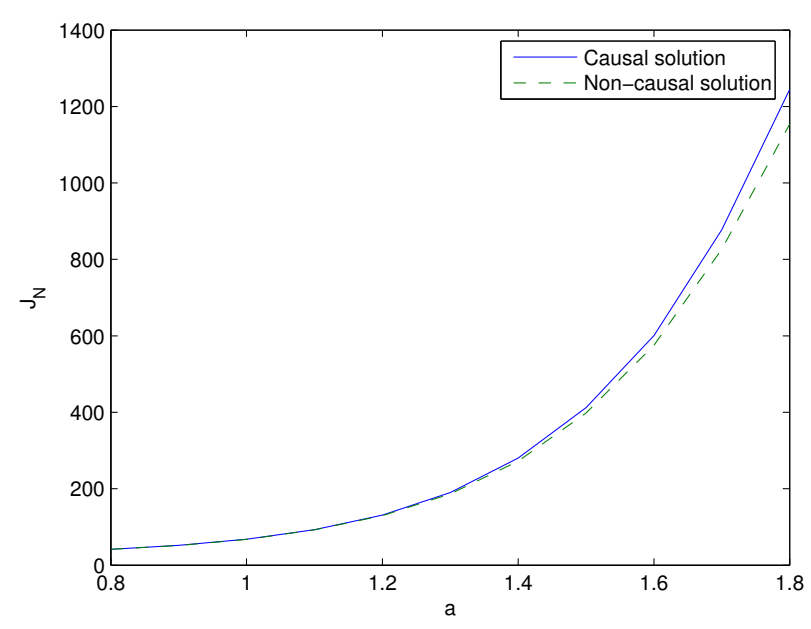

Fig. 2. Scalar system, finite horizon. Comparison between causal and non-causal control.

In Figure 3 we plot the infinite horizon cost $J_{\infty}$ for the infinite horizon solution with full CSI (13)-(14), and infinite horizon solution with statistical CSI of Section 4, for different values of $a$. From numerical computation, we find that for values of a greater than around 1.35, the stability criteria is no longer satisfied in the case with statistical CSI and the infinite horizon cost diverges. 


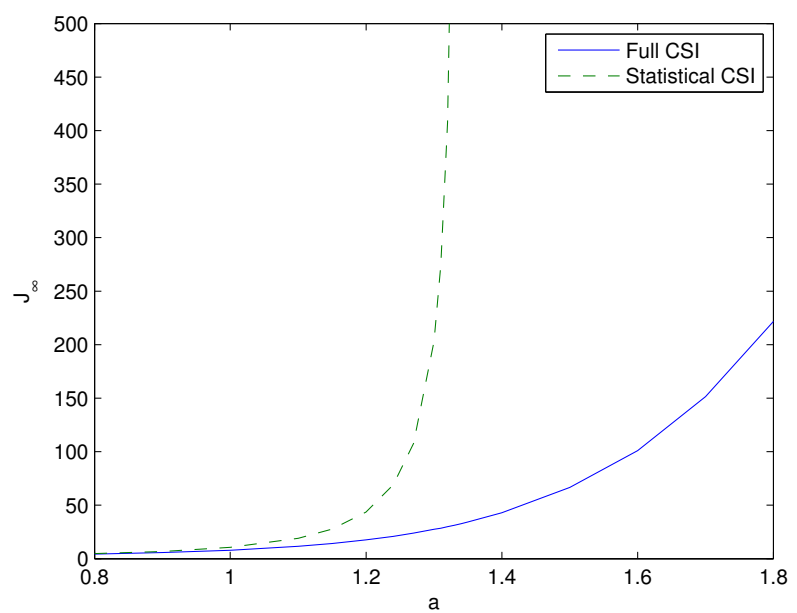

Fig. 3. Scalar system, infinite horizon. Comparison between full CSI and statistical CSI solutions

\section{CONCLUSION}

In this paper we have considered the optimal control of a system where there are continuous valued fading channels between the sensor and controller, and between the controller and actuator. We have derived the optimal LQG controller under full CSI and statistical CSI assumptions. Future work will include jointly optimizing the powers used in transmitting the sensor measurements and control signals over the fading channels, by optimizing the choices of the amplification factors $\alpha_{k}$ and $\beta_{k}$.

\section{REFERENCES}

Anderson, B.D.O. and Moore, J.B. (1979). Optimal Filtering. Prentice Hall, New Jersey.

Bertsekas, D.P. (2000). Dynamic Programming and Optimal Control, Volume I. Athena Scientific, Belmont, Massachusetts, 2nd edition.

Caire, G., Taricco, G., and Biglieri, E. (1999). Optimum power control over fading channels. IEEE Trans. Inform. Theory, 45(5), 1468-1489.

Chizeck, H.J. and Ji, Y. (1988). Optimal quadratic control of jump linear systems with Gaussian noise in discretetime. In Proc. IEEE Conf. Decision and Control, 19891993. Austin, Texas.

Cui, S., Xiao, J.J., Goldsmith, A., Luo, Z.Q., and Poor, H.V. (2007). Estimation diversity and energy efficiency in distributed sensing. IEEE Trans. Signal Processing, 55(9), 4683-4695.

De Koning, W.L. (1992). Compensatability and optimal compensation of systems with white parameters. IEEE Trans. Automat. Contr., 37(5), 579-588.

Dey, S., Leong, A.S., and Evans, J.S. (2009). Kalman filtering with faded measurements. Automatica, 45(10), 2223-2233.

Epstein, M., Shi, L., Tiwari, A., and Murray, R.M. (2008). Probabilistic performance of state estimation across a lossy network. Automatica, 44, 3046-3053.

Gastpar, M. and Vetterli, M. (2003). Source-channel communication in sensor networks. Springer Lecture Notes in Computer Science, 2634, 162-177.
Gupta, V., Hassibi, B., and Murray, R.M. (2007). Optimal LQG control across packet-dropping links. Systems and Control Letters, 56, 439-446.

Horn, R.A. and Johnson, C.R. (1991). Topics in Matrix Analysis. Cambridge University Press, Cambridge, United Kingdom.

Huang, M. and Dey, S. (2007). Stability of Kalman filtering with Markovian packet losses. Automatica, 43, 598-607.

Imer, O.C., Yüksel, S., and Başar, T. (2006). Optimal control of LTI systems over unreliable communication links. Automatica, 42, 1429-1439.

Mostofi, Y. and Murray, R.M. (2005). On dropping noisy packets in Kalman filtering over a wireless fading channel. In Proc. American Control Conf., 4596-4600. Portland, OR.

Schenato, L. (2006). Optimal estimation in networked control systems subject to random delay and packet drop. In Proc. IEEE Conf. Decision and Control. San Diego, CA.

Schenato, L., Sinopoli, B., Franceschetti, M., Poolla, K., and Sastry, S.S. (2007). Foundations of control and estimation over lossy networks. Proc. IEEE, 95(1), 163187.

Sinopoli, B., Schenato, L., Franceschetti, M., Poolla, K., Jordan, M.I., and Sastry, S.S. (2004). Kalman filtering with intermittent observations. IEEE Trans. Automat. Contr., 49(9), 1453-1464.

Sinopoli, B., Schenato, L., Franceschetti, M., Poolla, K., and Sastry, S. (2006). Optimal linear LQG control over lossy networks without packet acknowledgment. In Proc. IEEE Conf. Decision and Control, 392-397. San Diego, CA.

Sinopoli, B., Schenato, L., Franceschetti, M., Poolla, K., and Sastry, S.S. (2005). Optimal control with unreliable communication: the TCP case. In Proc. American Control Conf., 3354-3359. Portland, OR.

$\mathrm{Xu}$, Y. and Hespanha, J.P. (2005). Estimation under uncontrolled and controlled communications in networked control systems. In Proc. IEEE Conf. Decision and Control, 842-847. Seville, Spain. 